\title{
Beryllium in turnoff stars of NGC 6397: Early Galaxy spallation, cosmochronology and cluster formation $\star, \star \star$
}

\author{
L. Pasquini ${ }^{1}$, P. Bonifacio ${ }^{2}$, S. Randich ${ }^{3}$, D. Galli ${ }^{3}$, and R. G. Gratton ${ }^{4}$ \\ ${ }^{1}$ European Southern Observatory, Garching bei München, Germany \\ e-mail: 1pasquin@eso.org \\ 2 INAF - Osservatorio di Trieste, Trieste, Italy \\ 3 INAF - Osservatorio di Arcetri, Firenze, Italy \\ ${ }^{4}$ INAF - Osservatorio di Padova, Padova, Italy
}

Received 7 May 2004 / Accepted 6 July 2004

\begin{abstract}
We present the first detection of beryllium in two turnoff stars of the old, metal-poor globular cluster NGC 6397. The beryllium lines are clearly detected and we determine a mean beryllium abundance of $\log (\mathrm{Be} / \mathrm{H})=-12.35 \pm 0.2$. The beryllium abundance is very similar to that of field stars of similar Fe content. We interpret the beryllium abundance observed as the result of primary spallation of cosmic rays acting on a Galactic scale, showing that beryllium can be used as a powerful cosmochronometer for the first stellar generations. With this method, we estimate that the cluster formed 0.2-0.3 Gyr after the onset of star formation in the Galaxy, in excellent agreement with the age derived from main sequence fitting. From the same spectra we also find low $\mathrm{O}$ (noticeably different for the two stars) and high $\mathrm{N}$ abundances, suggesting that the original gas was enriched in $\mathrm{CNO}$ processed material. Our beryllium results, together with the $\mathrm{N}, \mathrm{O}$, and $\mathrm{Li}$ abundances, provide insights on the formation of this globular cluster, showing that any CNO processing of the gas must have occurred in the protocluster cloud before the formation of the stars we observe now. We encounter, however, difficulties in giving a fully consistent picture of the cluster formation, able to explain the complex overall abundance pattern.
\end{abstract}

Key words. stars: abundances - Galaxy: globular clusters: individual: NGC 6397 - stars: formation - ISM: cosmic rays stars: late-type

\section{Introduction}

Beryllium has a unique origin: it is produced in the interstellar medium (ISM) by Galactic cosmic rays (GCRs) through the spallation of carbon, oxygen and nitrogen nuclei (Reeves et al. 1970); unlike lithium it is not produced during the Big Bang, unlike most other elements is not produced in stars. It is well established that ${ }^{6} \mathrm{Li}$, beryllium and $\mathrm{B}$ are produced in the Galactic halo by two spallative processes: (i) collisions of accelerated $\mathrm{C}, \mathrm{N}, \mathrm{O}$ nuclei in GCRs with ISM protons and $\alpha$ particles (a primary process leading to a linear dependence of $\mathrm{Be}$ on metallicity); (ii) collisions of energetic protons and $\alpha$ particles in GCRs with ISM C,N,O nuclei (a secondary process leading to a quadratic dependence of beryllium on metallicity) ${ }^{1}$. The primary process (i) affects the Galaxy as a whole and is predicted to dominate the production of beryllium in

\footnotetext{
* Based on observations collected at the ESO VLT, Paranal Observatory, Chile.

$\star \star$ Table 2 is only available in electronic form at http://www.edpsciences.org

${ }^{1}$ In addition, ${ }^{6,7} \mathrm{Li}$ can be produced also by fusion $(\alpha+\alpha)$ reactions in the ISM, a process important for the production of $\mathrm{Li}$ isotopes in a metal-poor gas.
}

the metal-poor ISM of the early Galaxy (Suzuki et al. 1999; Suzuki \& Yoshii 2001). The spallative origin of beryllium implies a power-law relation between the abundance of beryllium and the metal abundance of stars, a trend confirmed observationally down to $[\mathrm{Fe} / \mathrm{H}] \simeq-3$ in metal-poor field halo stars (Boesgaard et al. 1999, hereafter B99). If, however, one is interested in the earliest phases of Galactic evolution, metallicity is no longer a reliable age indicator. As a consequence of the dispersed character of star formation in the Galactic halo and the lack of efficient mixing in the gas, the chemical composition of the ISM in the first stages of Galactic evolution was strongly affected by local enrichment produced by individual type II supernovae. Observations of very metal metal poor stars (Ryan et al. 1996; McWilliam 1997) show in fact a large, probably intrinsic, spread of elemental abundances, interpreted as an indication of inhomogeneous chemical evolution of the early Galaxy (e.g., Travaglio et al. 2001; Suzuki \& Yoshii 2001). Even if the most recent, high quality data show that part of this spread is due to the limited quality of the previous data (Cayrel et al. 2004), a one-to-one relation of metallicity with age in the first stellar generations is not expected. Conversely, beryllium, B and the isotope ${ }^{6} \mathrm{Li}$, being produced 
by energetic particles generated and transported globally on a Galactic scale, are expected to show a much smaller spread of abundances than the products of type II supernovae (typically oxygen), making them ideal "cosmic clocks" for dating the first stages of halo evolution (Suzuki et al. 2001; Beers et al. 2000). Globular clusters represent ideal test cases for this new clock because they are an extremely old stellar population for which independent, reliable age determinations are possible (Salaris \& Weiss 2002; Gratton et al. 2003, hereafter G03).

To test beryllium as a cosmic clock it is necessary to measure beryllium in stars which can be dated independently. Stars in old globular clusters such as NGC 6397 are very good candidates, because their ages can be determined in a reliable way and they have been shown to be formed within $\sim 1$ Gyr after the Big Bang (G03). However, the search for beryllium in metalpoor stars has been limited so far to relatively bright field stars $(V \leq 12.5)$, because the only available beryllium lines are the Be II resonance doublet at $313.1 \mathrm{~nm}$. This wavelength is very close to the atmospheric cut-off at $300 \mathrm{~nm}$ and the terrestrial atmosphere heavily absorbs the incoming radiation, making observations very challenging. In addition, the equivalent width of the Be lines is very small and the spectral region crowded, so that high spectral resolution is necessary.

The high UV efficiency of UVES at the VLT telescope Kueyen has opened a new possibility, allowing for the first time the detection of beryllium in two turn-off (TO) stars in NGC 6397. It is crucial to reach the cluster TO because these stars have not depleted beryllium in their atmospheres. Conversely, brighter subgiants in the same cluster show clear evidence of Li dilution (Castilho et al. 2000, hereafter C00). Since this more fragile element is at its original level in TO stars, then, a fortiori, beryllium is not expected to be depleted in their atmospheres.

\section{Basic properties of NGC 6397}

NGC 6397 is one of the closest and best studied globular clusters. In particular it has been the subject of several recent high resolution spectroscopic studies which have shown a very good agreement on the cluster $[\mathrm{Fe} / \mathrm{H}]$ abundance and on its homogeneity along the color-magnitude diagram. The cluster Fe abundance determined spectroscopically by several groups (see $\mathrm{C} 00$, and references therein), is in the range $-2.2<$ $[\mathrm{Fe} / \mathrm{H}]<-1.8$. The most recent determinations agree on the value $[\mathrm{Fe} / \mathrm{H}] \simeq-2.0(\mathrm{C} 00$; Thévenin et al. 2001 , hereafter T01; Gratton et al. 2001, hereafter G01), although these studies adopt different techniques: LTE analysis in C00 and G01, non-LTE computations in T01. A detailed chemical analysis of NGC 6397 has been carried out by T01, who found abundances of $\alpha$ - and Fe-peak elements in agreement with those of field stars of similar metallicity.

The Li abundance in TO stars of NGC $6397(\log (N(\mathrm{Li})=$ $2.36 \pm 0.05$, Molaro \& Pasquini 1994; C00; Bonifacio et al. 2002, hereafter B02) is consistent with the Spite's Li plateau (Spite \& Spite 1982). The Li abundance decreases with the star advancing on the red-giant branch (RGB) because of dilution, and only upper limits can be obtained for stars brighter than the RGB bump (C00). The average cluster $\mathrm{O}$ abundance
Table 1. NGC 6397 stars, their atmospheric parameters and abundances. The atmospheric parameters are from B02, while the abundances are from this work and T01.

\begin{tabular}{lllllll}
\hline \hline Star & $T_{\text {eff }}$ & $\log g$ & {$[\mathrm{Fe} / \mathrm{H}]$} & {$[\mathrm{N} / \mathrm{H}]$} & {$[\mathrm{O} / \mathrm{H}]$} & $\log (\mathrm{Be} / \mathrm{H})$ \\
\hline A0228 & 6274 & 4.1 & $-2.05^{a}$ & -0.74 & -2.24 & -12.27 \\
A2111 & 6207 & 4.1 & $-2.01^{a}$ & -0.74 & -1.64 & -12.43 \\
HD 218502 & $6296^{b}$ & 4.13 & $-1.85^{b}$ & $-1.95:$ & & -12.36 \\
\hline
\end{tabular}

(a) From T01.

${ }^{(b)}$ From Alonso et al. (1999).

appears to be lower than that of field stars of similar metallicity $([\mathrm{O} / \mathrm{Fe}] \simeq 0.2 \pm 0.1$ according to $\mathrm{C} 00$ and $\mathrm{G} 01)$, but variations of $\mathrm{O}$ are claimed among the subgiants, possibly anticorrelated with Na (Carretta et al. 2004). Thus, although NGC 6397 is more homogeneous than most globular clusters, it nevertheless shows signs of contamination by gas processed through CNO cycling.

\section{Sample selection and observations}

We selected from the T01 sample the two brightest stars to allow the challenging beryllium observations. The selected stars, together with their atmospheric parameters (B02) and chemical abundances (T01), are listed in Table 1.

The observations were carried out in service mode at the VLT observatory in several runs during summer 2003 with the UVES spectrograph (Dekker et al. 2000). We used a 1" slit providing a resolving power of 45000 . The blue CCD was binned along the spatial direction to minimize the CCD read out noise. We used the dichroic to obtain, in addition to the UV data, spectra with the red $860 \mathrm{~nm}$ setup, containing the $\mathrm{O}_{\mathrm{I}} 777 \mathrm{~nm}$ triplet which we used to derive the $\mathrm{O}$ abundances. A total of 8 exposures of 90 min each were obtained per star. The observations were reduced with the UVES pipeline (Ballester et al. 2000). The reduced spectra were averaged leading to a $S / N$ ratio in the beryllium region of 8 and 15 per pixel for star A2111 and A228 respectively. The spectra in the beryllium region, together with our synthetic best fits (cf. next section) are shown in Fig. 1. In addition to the cluster stars, we obtained high $S / N(\sim 130)$ spectra of the bright reference star HD 218502, whose atmosperic parameters are very close to the ones of the cluster stars (cf. Table 1).

\section{Data analysis: Stellar parameters and determination of abundances}

Table 1 lists the adopted atmospheric stellar parameter (B02) and the abundance values determined in this work.

A full synthesis of the region around the beryllium doublet has been performed using the line list given in Table 2 . In this table the ions are identified by their code, according to Kurucz's convention (Kurucz 1993). Almost all lines have been extracted by a version of the Kurucz data base updated by F. Castelli (private communication). The only exception are the $\mathrm{OH}$ lines, for which gf values have been computed from lifetimes of Goldman \& Gillis (1981). We used our Linux version (Sbordone et al. 2004) of the SYNTHE code 


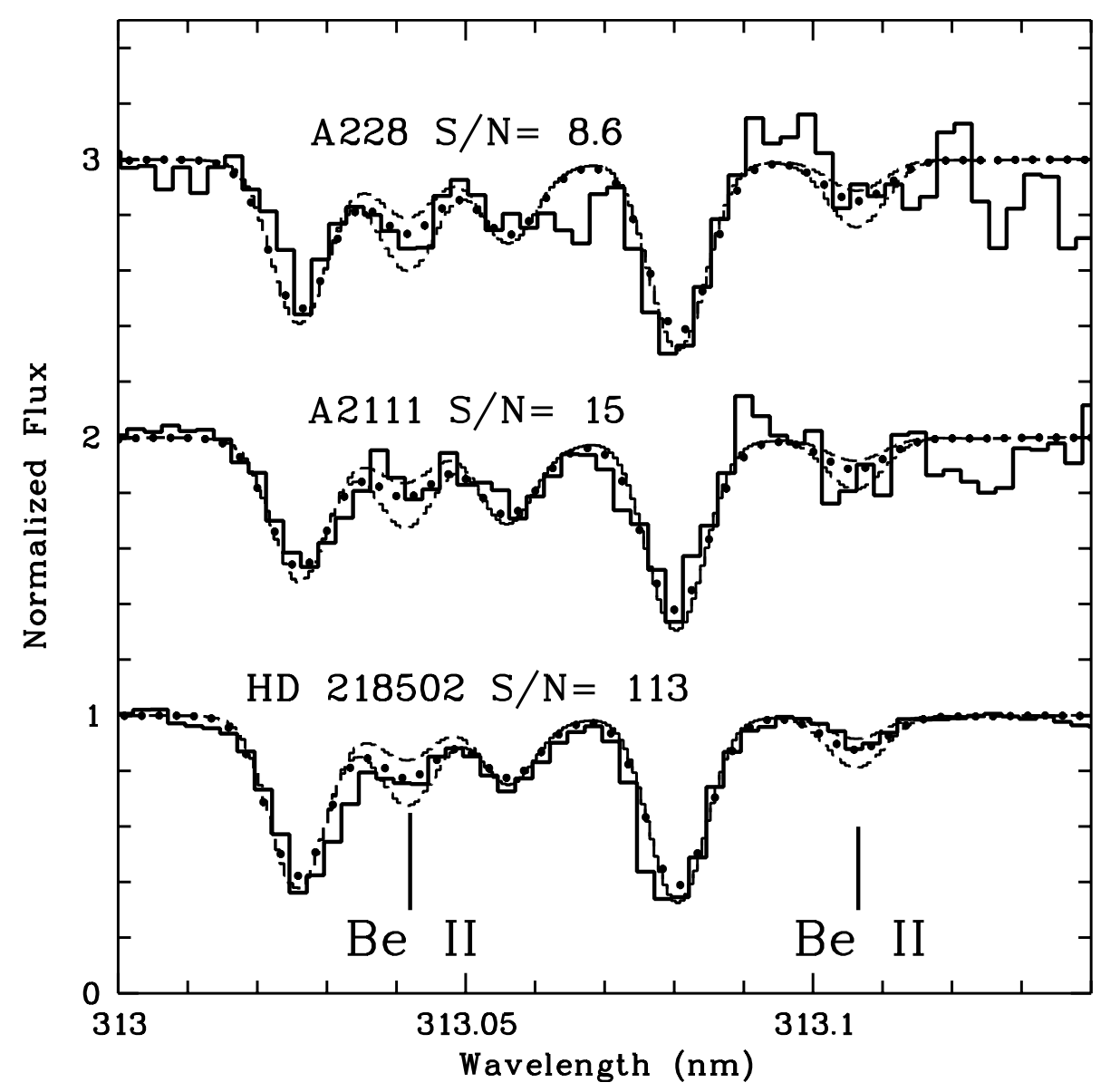

Fig. 1. UVES spectra of the two turn-off stars A228 and A2111 of the globular cluster NGC 6397. The dotted lines correspond to the bestfit Be abundances $\log (\mathrm{Be} / \mathrm{H})=-12.27$ for $\mathrm{A} 228$ and $\log (\mathrm{Be} / \mathrm{H})=-12.43$ for $\mathrm{A} 2111$. The dashed lines correspond to synthetic spectra with abundances of \pm 0.2 dex from the best fitting abundance. For comparison, we also show the spectrum of the bright halo star HD 218502, whose atmospheric parameters are close to those of the NGC 6397 TO stars. The best fit synthetic spectrum for this star corresponds to $\log (\mathrm{Be} / \mathrm{H})=-12.36$.

(Kurucz 1993). For each star the best abundance was found by a $\chi^{2}$ fit to the whole feature. The best fits, shown in Fig. 1, correspond to beryllium abundances of $\log (\mathrm{Be} / \mathrm{H})=-12.27$, and $(\mathrm{Be} / \mathrm{H})=-12.43$ for $\mathrm{A} 228$ and $\mathrm{A} 2111$, respectively. For HD 218502, we derive an abundance of $\log (\mathrm{Be} / \mathrm{H})=-12.36 \pm$ 0.1 , in agreement with the previous determination by Molaro et al. (1997), $\log (\mathrm{Be} / \mathrm{H})=-12.56 \pm 0.22$.

We estimate the errors with Monte Carlo simulations assuming a Poisson noise. The dispersion around the mean in 1000 Monte Carlo samples was 0.09 dex for A2111 and 0.11 dex for A228. These should be viewed as lower limits on the errors, since at these low $S / N$ ratios other sources of nonPoisson noise (e.g. shot-noise) could be important. To these errors associated to the noise in the data one must add the errors which derive from the uncertainty in the atmospheric parameters. Since we are using lines of singly ionized beryllium, surface gravity is the parameter which most affects the beryllium abundance computation. The values of $\log g$ have been estimated from the position of the stars in the color-magnitude diagram and theoretical isochrones. Since the uncertainty in the derivation of gravity in the case of these clusters stars is dominated by the unceratinty in their mass, we can safely assume an error of $0.15 \mathrm{dex}$ in $\log g$ that translates into an uncertainty in the beryllium abundance of 0.08 dex. The error arising from a change of $100 \mathrm{~K}$ in the effective temperature is also 0.08 dex. Summing these two errors under quadrature one obtains $0.11 \mathrm{dex}$. If we add in quadrature this value to the errors due to noise ( 0.09 dex and 0.11 dex, respectively), we obtain an estimate of the total error on the beryllium abundance of 0.14 dex for A2111 and 0.15 dex for A228. Therefore we conclude that the beryllium abundance of the two stars is the same within the errors. We can then estimate the average beryllium abundance of NGC 6397 as $\log (\mathrm{Be} / \mathrm{H})=-12.35 \pm(0.10)_{\text {stat }} \pm$ $(0.11)_{\text {sys. }}$ The systematic error is estimated as due to an uncertainty of the zero point of the temperature scale by $100 \mathrm{~K}$ and an uncertainty of the zero point of the $\log g$ scale by 0.15 dex. Note that this error does not include possible systematic errors due to shortcomings and inadequacies of our modelling (model atmospheres, atomic data, etc.).

The oxygen abundance has been computed from the infrared triplet lines, using the same method as G01. In Fig. 2 we show the spectra of the two NGC 6397 stars; the triplet lines are very weak, and in particular for A228 they are at the limit of detectability. Considering that the two cluster stars have similar stellar parameters, Fig. 2 suggests that the two stars have different $\mathrm{O}$ abundances. In fact we derive $[\mathrm{O} / \mathrm{H}]=-1.64 \pm 0.05$ 

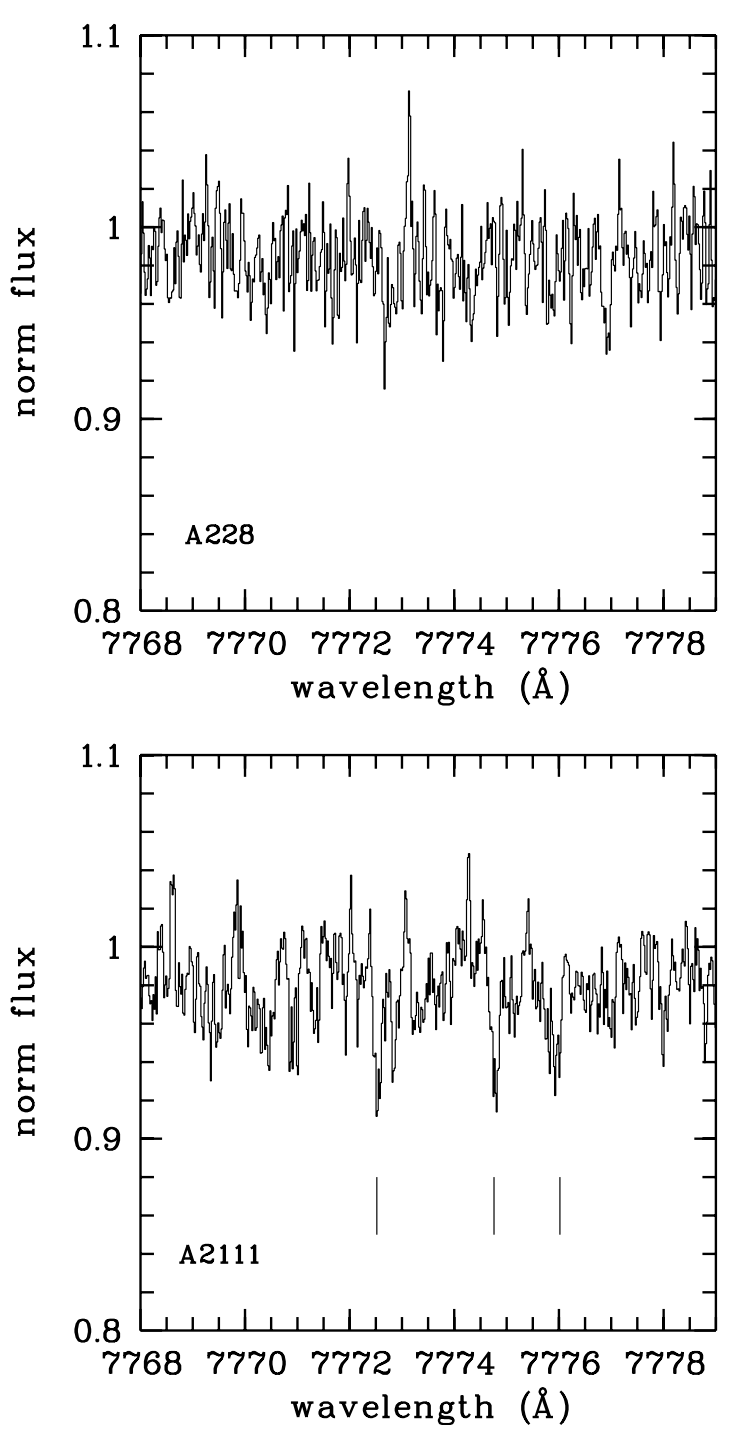

Fig. 2. UVES spectra of stars A2111 and A228 in the oxygen triplet region. The two stars have identical atmospheric parameters, but the $\mathrm{O}$ abundance differs almost by 0.6 dex.

and $[\mathrm{O} / \mathrm{H}]=-2.24 \pm 0.15$ for A2111 and A228, respectively. Previous studies of O in stars of NGC 6397 have obtained low $\mathrm{O}$ abundances $([\mathrm{O} / \mathrm{Fe}] \simeq 0.2$ according to $\mathrm{C} 00$ and $\mathrm{G} 01)$ with respect to stars of similar metallicity. Carretta et al. (2004) have shown that a spread in $\mathrm{O}$ abundance is present among the subgiants of this cluster. For the first time evidence of $\mathrm{O}$ dispersion is also found in main sequence stars of NGC 6397.

We have also been able to measure the $\mathrm{N}$ abundance in these two cluster stars, confirming that the TO stars are $\mathrm{N}$-rich, like the subgiant stars studied by Carretta et al. (2004). Nitrogen is a difficult element to measure, especially for dwarf stars, since the NI lines become extremely weak at moderately low metallicity. On the other hand, $\mathrm{CN}$ bands, which can be used down to very low metallicity in giant and subgiant stars are not measurable in dwarfs. At low metallicities the only feature which has been used to measure $\mathrm{N}$ is the $\mathrm{NH}$ band at $336 \mathrm{~nm}$, which is very near to the atmospheric cut-off. Among field stars the $[\mathrm{N} / \mathrm{Fe}]$ ratio is approximately constant with metallicity in the range $-3.0 \leq[\mathrm{Fe} / \mathrm{H}] \leq 0.4$,
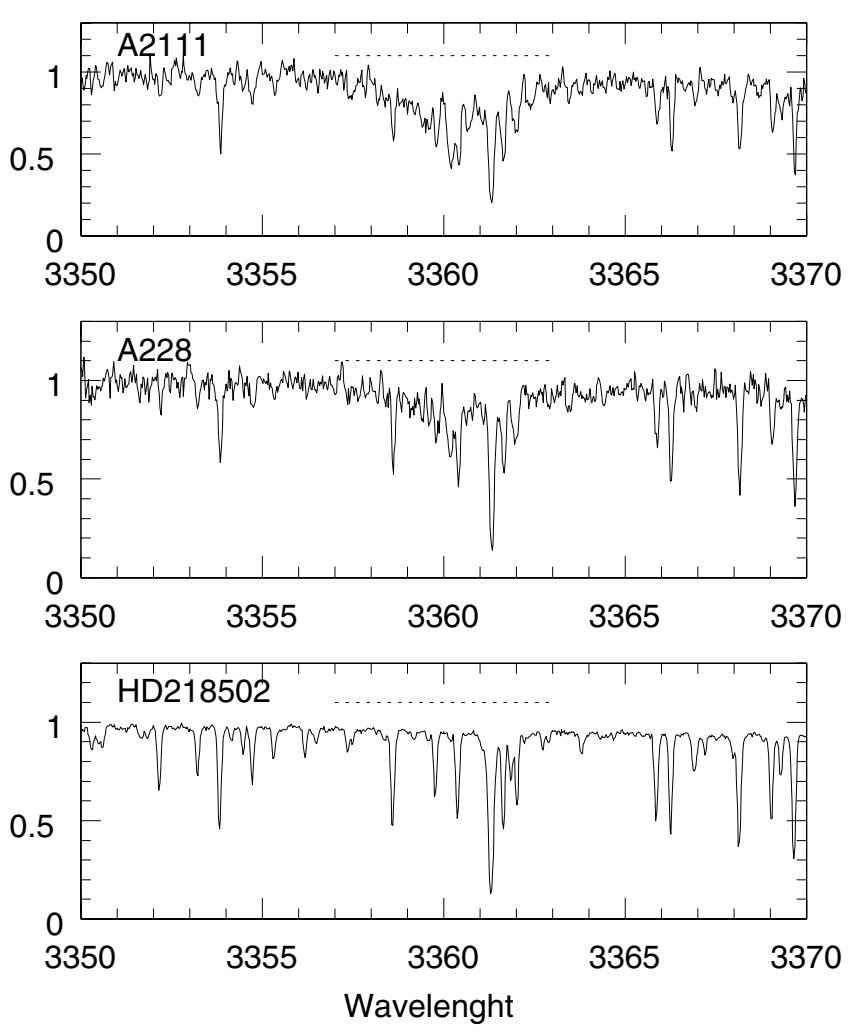

Fig. 3. UVES spectra of stars A2111 and A228 around the NH band at $336 \mathrm{~nm}$. For comparison, we also show the same spectral region for our reference star HD 218502, whose atmospheric parameters $\left(T_{\text {eff }}=6296 \mathrm{~K}, \log g=4.13,[\mathrm{Fe} / \mathrm{H}]=-1.85\right)$ are close to those of the NGC 6397 TO stars. While the band (whose core region is indicated by the dashed lines) is virtually absent in the reference star, it is well pronounced in the two cluster stars, indicating a strong $\mathrm{N}$ overabundance.

as first shown by Carbon et al. (1987) and recently confirmed by Israelian et al. (2004) and Ecuvillon et al. (2004) on the basis of higher quality data. We notice however the existence in these samples of a non-negligible population of N-rich stars, i.e. stars which have a $\mathrm{N}$ abundance higher than most stars of similar metallicity. The presence of a few N-rich stars has been known for a long time (Bessell \& Norris 1982; Laird 1985) but their census is steadily increasing.

We estimate the nitrogen abundance by spectral synthesis of two NH UV bands, around $336 \mathrm{~nm}$ and around $340.5 \mathrm{~nm}$. Given the complexity of the bands it is not possible to carry out a formal fitting procedure of the whole spectral region. We have therefore inferred a "best abundance" by fitting the most prominent features. Despite several systematic and random uncertainties, we are able to confirm the overabundance of $\mathrm{N}$ in both NGC 6397 TO stars with respect to N-normal field stars of similar metallicity. In Fig. 3 we compare the NH bands of the two NGC 6397 stars and of HD 218502, in order to emphasize the N-rich nature of the cluster stars. 


\section{Implications on Galactic chemical evolution and cluster formation}

In this section we discuss the theoretical implications of our beryllium determination in NCG 6397. We first show how the beryllium abundance in halo stars can be used as a powerful cosmochronometer, due to the fact that spallation in the early Galaxy was dominated by primary fragmentation on a Galactic scale. We then discuss the complex abundance pattern observed in NGC 6397 and its implications on cluster formation scenarios.

\subsection{Beryllium vs. oxygen in cluster and halo stars}

As mentioned in the Introduction, cosmic-ray spallation in the early Galaxy was dominated by primary reactions, where fast, heavy (mostly CNO) nuclei in the cosmic rays are broken by encounters with ISM protons and $\alpha$ particles. This process predicts a linear dependence of beryllium with metallicity in the whole Galaxy, as well as an approximately linear increase of beryllium with time. One of the most important characteristics is that, being dominated by a global production mechanism, the beryllium abundance is expected to be largely independent of the local chemical inhomogeneities which may have been present in the early halo (Beers et al. 2000; Suzuki et al. 1999). On the other hand, the cosmic ray flux is driven by the global star formation rate and to a certain extent by the confining mechanism, which were likely related to the large scale magnetic fields, and our knowledge of all these early quantities is very limited.

The contribution of secondary spallation processes (collisions of accelerated protons and $\alpha$ particles with heavy nuclei in the ISM) is not dominant at these early epochs, and therefore the observed underabundance of $\mathrm{O}$ in the cluster stars should not have dramatically affected the beryllium abundance. In any case, since the beryllium production factors from spallation of C, N, O nuclei are very similar (cf. e.g., Valle et al. 2002), $\mathrm{C}$ or $\mathrm{N}$ overabundances may compensate an $\mathrm{O}$ deficit and result in a comparable amount of beryllium as from a "standard" mixture.

The beryllium abundance of the NGC 6397 stars is fully consistent with the well-known Be vs. Fe trend (Gilmore et al. 1992; Molaro et al. 1997; B99). In Fig. 4 we show the Be vs. O relation for the field stars from B99, and the NGC 6397 values determined in this work. Although the $\mathrm{O}$ determination is carried out in different ways in our and Boesgaard's study, a comparison of 13 stars in common with G03 shows an excellent agreement between the two scales. As Fig. 4 shows, the cluster stars lie above the mean Be vs. O trend of field halo stars, as expected since they have lower $[\mathrm{O} / \mathrm{Fe}]$ than field stars with the same Fe content. In particular, Fig. 4 shows that whereas A2111 is marginally consistent with the general Be vs. O trend in field stars, A228 is strongly oxygen-poor given its beryllium content. This discrepancy can be understood if primary spallation mechanisms dominate the beryllium production in the early Galactic phases. In that case, the amount of beryllium produced depends more on the cosmic-ray composition than on the local composition of the protocluster gas.

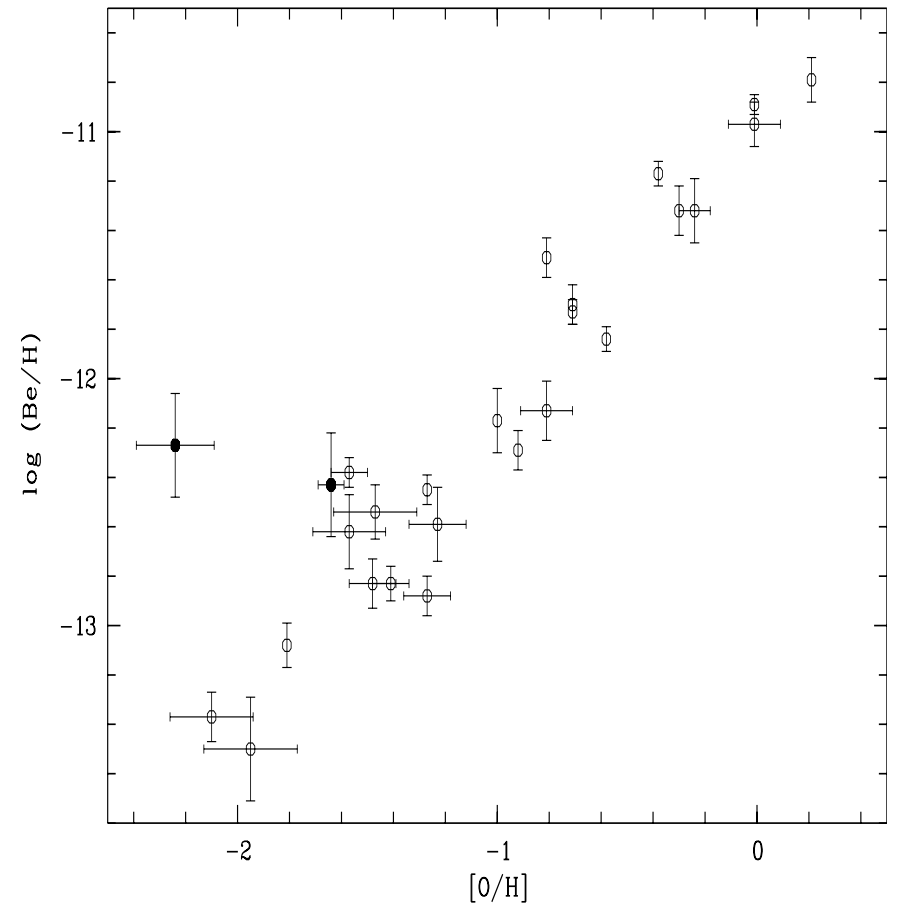

Fig. 4. Be abundance vs. oxygen for the NGC 6397 TO stars (filled circles) and the star from B99, (open circles).

\subsection{Be as cosmochronometer and the age of NGC 6397}

NGC 6397 is an ideal target to prove the usefulness of beryllium as a cosmic clock, as outlined in the Introduction. This cluster has been independently dated via main-sequence fitting and theoretical isochrones (G03) and its TO stars are bright enough to allow detection of beryllium in their atmospheres. Using standard isochrones, G03 find that the age of the cluster is $13.9 \pm 0.4$ Gyr. Taking into account a small amount of gravitational settling in the stellar tracks, reduces the age of the cluster by about $0.5 \mathrm{Gyr}$. Therefore, the best age estimate for this cluster is $13.4 \pm 0.8 \pm 0.6$ Gyr (G03).

Figure 5 shows the expected evolution of beryllium with time, resulting from a model of chemical evolution (Valle et al. 2003) following the enrichement of three different regions in the Galaxy, coupled by mass flows: the halo, the thick disk and the thin disk. The beryllium abundance of the model is normalized to the solar meteoritic value, assuming that the solar system formed 4.5 Gyr ago. The other data points in Fig. 5 shows the beryllium abundance in the young open cluster IC 2391 (Randich et al. 2002), as indicator of the present day value, and the average value of the two stars of NGC 6397 , determined in this paper. We have assumed a Galactic age of 13.7 Gyr, based on WMAP data (Bennett et al. 2003). This value represents the "age of the Universe" (time elapsed since the Big Bang), whereas the actual Galactic age (time elapsed since the onset of star formation in the Galaxy) should take into account the time interval between the Big Bang and the epoch of reionization. The best estimate for this interval, according to WMAP data, is $\sim 0.18 \mathrm{Gyr}$, corresponding to $z_{\text {reion }}=20$, within the errors on the age estimate of NGC 6397. 


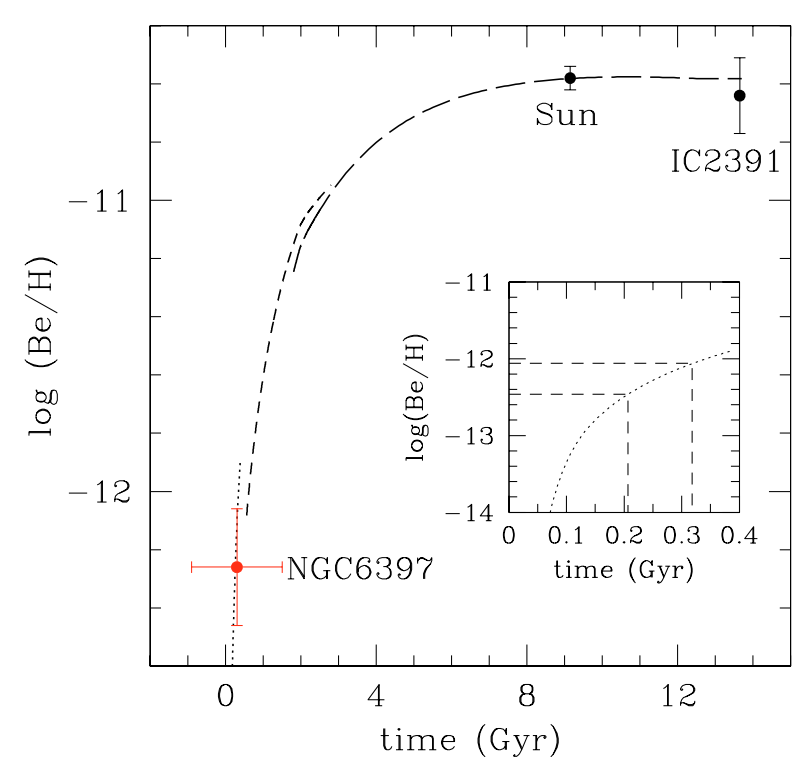

Fig. 5. Evolution of beryllium with time in the Galaxy according to a three-zone Galactic chemical evolution model (Valle et al. 2002). The three curves refer to the halo, thick disk, and thin disk. The data points show the beryllium abundance in the young open cluster IC 2391 (age $30 \mathrm{Myr}$ ), the Sun (age 4.5 Gyr), and the globular cluster NGC 6397. The model result is normalized to the solar meteoritic abundance. The inset illustrate the use of beryllium as a "cosmic clock" to constrain the formation of NGC 6397. The horizontal lines corresponds to the $1 \sigma$ counturs around the measured beryllium abundance. The cluster birth is constrained to the first $0.2-0.3 \mathrm{Gyr}$ after the onset of star formation in the Galactic halo.

The inset in Fig. 5 shows the halo evolution of beryllium on a finer scale, with the two horizontal lines limiting the $1 \sigma$ range of beryllium abundance in NGC 6397. This plot emphasizes the possible use of beryllium as "cosmic clock": the measured value of beryllium indicates that the formation of NGC 6397 occurred about 0.2-0.3 Gyr after the onset of star formation in the Galactic halo. Considering all the uncertainties present in the model it is safe to conclude that the birth of the stars composing NGC 6397 took place within the first $\sim 0.5$ Gyr of the halo evolution, in agreement with the isochrone fitting age determination for this cluster. We stress the fact that the "Be age" of the cluster is a measure of the time interval between the onset of star formation in the Galaxy and the formation of the cluster, whereas the "evolutionary age" of NGC 6397 is a measure of the time elapsed since the formation of the cluster and the present epoch. The agreement of the two methods indicated a global consistency of the results of stellar evolution, cosmicray nucleosynthesis and cosmology. This result is largely independent from the chemical model adopted, because for the early phases of Galactic evolution the beryllium production rates between different models agree to within better than a factor 2, which is comparable to the uncertainties of the beryllium determination in NGC 6397.

The "beryllium age" method can be extended in principle to field halo stars for which independent age determinations are difficult (see e.g., Beers et al. 2000). We note that according to this approach, and under the hypothesis that the non-zero metallicity field stars we observe today formed several Myr after the onset of the star formation in the halo, then their beryllium should be at low, but non-negligible levels of abundance (e.g. $\log (\mathrm{Be} / \mathrm{H}) \simeq 13.6$ for $100 \mathrm{Myr})$. On the other hand their metal abundances should be sensitive to local enrichment phenomena, thus stars of the same age and beryllium abundance could show different $[\mathrm{Fe} / \mathrm{H}]$ and $[\mathrm{O} / \mathrm{H}]$. The beryllium-metallicity relationship may therefore present some considerable scatter at the lowest metallicity end, as possibly indicated by recent observations of extremely metal poor stars (B99; Primas et al. 2000).

\subsection{The formation of NGC 6397}

As we have already discussed in Sect. 4, recent observations of NGC 6397, as well as our own results, strongly indicate an oxygen underabundance with respect to field stars with similar Fe (C00; G01), a nitrogen overabundance, and a star-tostar scatter in oxygen abundance (Carretta et al. 2004). Other $\alpha$ and Fe-peak elements (like $\mathrm{Mg}, \mathrm{Na}, \mathrm{Ca}, \mathrm{Sc}, \mathrm{Ti}, \mathrm{Cr}, \mathrm{Ni}, \mathrm{Zn}$, and $\mathrm{Ba}$ ), however, do not show significant star-to-star variations, and are consistent with those of field halo stars of similar metallicity (G01; T01). The low $\mathrm{O}$ and high $\mathrm{N}$ abundances suggest that the stars of NGC 6397 were either formed from, or were partially polluted by, material bearing the signature of asymptotic giant-branch (AGB) products (Chieffi et al. 2001; Ventura et al. 2002). In this phase, stellar material is processed at the very high temperatures $\left(\sim 10^{8} \mathrm{~K}\right)$ where $\mathrm{O}$ is effectively burnt to $\mathrm{N}$ in the CNO cycle and then returned to the ISM by mass loss. However, at the high temperatures characterizing AGB nucleosynthesis, Li and beryllium are completely destroyed. It is therefore quite challenging to explain the normal (Pop II) level of Li observed in NGC 6397 (B02). In principle, Li could be produced during the AGB phase and brought to the surface through the Cameron-Fowler mechanism (Cameron \& Fowler 1971; Sackmann \& Boothroyd 1992). However, a remarkable fine tuning between $\mathrm{Li}$ production and destruction is needed to bring at the surface of these AGB stars (and into the ISM) an amount of Li exactly equal to the primordial value (see discussion in B02, and references therein). Another weak point of this scenario, is that according to our observations, at least some of the low-mass stars of NGC 6397 should have been formed from material partially polluted by a previous generation of AGB stars. Just as an example, if we assume that the star A2111 is representative of an unpolluted star, the O abundance of star A228 indicates that $3 / 4$ of its mass were polluted by AGB ejecta. In addition, the high $\mathrm{N}$ content of star A2111 (which is supposely an example of "unpolluted" object) will remain unexplained. The presence of beryllium, which is only destroyed during the AGB phase, and is not produced by stellar nucleosynthesis, makes the possibility that a considerable fraction of the gas of the protocluster was processed by a previous generation of AGB stars quite unlikely.

One could image an AGB scenario where the gas was processed extremely early in the AGB stars and immediately released to the ISM, where it was exposed to the GCRs, but it seems difficult to satisfy all the previous points. Assuming a different perspective, one could argue that $\mathrm{N}$ rich gas is 
primarily produced in massive stars and then that $\mathrm{N}$-rich, $\mathrm{H}$-poor gas is released to the ISM (see e.g., Meynet \& Maeder 2002, for possible yelds). While this scenario could explain the $\mathrm{N}$ overabundance, it would require some additional mechanism to explain the origin of the $\mathrm{O}$ underabundance.

We notice the similarity of the timescales involved in the different enrichment processes probably at work in these early phase of halo evolution. While the lifetime of a $5 M_{\odot}$ star (a typical AGB progenitor) of zero metallicity is $\sim 0.1 \mathrm{Gyr}$ (Chieffi et al. 2001), our model of Galactic evolution shows that an irradiation timescale of $\sim 0.2$ Gyr is required to bring the beryllium abundance up to the observed values. According to our model, a similar timescale is also necessary to raise the iron content of the gas from zero to $[\mathrm{Fe} / \mathrm{H}] \simeq-2$. Thus, the emerging picture seems to indicate the simultaneous presence in the early Galaxy of both local (type II supernovae, AGB stars) and global (GCRs) enrichment processes, acting on comparable timescales during the rapid phase of star formation in the halo.

We are therefore left without a satisfying solution to the overall abundance pattern observed in NGC 6397. Our results on beryllium, however, imply that the TO stars we observe today did not process material in their interiors and their chemical composition is representative of the primordial protocluster gas.

Acknowledgements. We thank A. Renzini and J. Walsh for several comments and suggestions. S.R. and D.G. acknowledge financial support by the Italian Ministero dell'Istruzione, dell'Università e della Ricerca through a COFIN grant. Finally, we would like to thank the UVES team for building such a wonderful instrument.

\section{References}

Ballester, P., Modigliani, A., et al. 2000, The Messenger 101, 31

Beers, T. C., Suzuki, T. K., \& Yoshii, Y. 2000, in The Light Elements and Their Evolution, ed. L. da Silva, M. Spite, \& J. R. de Medeiros, PASP, IAU Symp., 198, 425

Bennett, C. L., Halpern, M., Hinshaw, G., et al. 2003, ApJS, 148, 1

Bessell, M. S., \& Norris, J. 1982, ApJ, 263, L29

Boesgaard, A. M., Deliyannis, C. P., King, J. R., et al. 1999, AJ, 117, 1549 (B99)

Bonifacio, P., Pasquini, L., Spite, F., et al. 2002, A\&A, 390, 91 (B02)
Cameron, A. G. W., \& Fowler, W. A. 1971, ApJ, 164, 111

Carbon, D. F., Barbuy, B., Kraft, R. P., Friel, E. D., \& Suntzeff, N. B. 1987, PASP, 99, 335

Carretta, E., et al. 2004 , in preparation

Castilho, B. V., Pasquini, L., Allen, D. M., Barbuy, B., \& Molaro, P. 2000, A\&A, 361, 92 (C00)

Cayrel, R., Depagne, E., Spite, M., et al. 2004, A\&A, 416, 1117

Chieffi, A., Domínguez, I., Limongi, M., \& Straniero, O. 2001, ApJ, 554,1159

Dekker, H., D’ Odorico, S., Kaufer, A., Delabre, B., \& Kotzlowski, H. 2000, SPIE, 4008, 534

Ecuvillon, A., Israelian, G., Santos, N. C., et al. 2004, A\&A, 418, 703

Gilmore, G., Gustafsson, B., Edvardsson, B., \& Nissen, P. E. 1992, Nature, 357, 379

Goldman, A., \& Gillis, J. R. 1981, JQSRT, 25, 111

Gratton, R. G., Bonifacio, P., Bragaglia, A., et al. 2001, A\&A, 369, 87 (G01)

Gratton, R. G., Bragaglia, A., Carretta, E., et al. 2003, A\&A, 408, 529 (G03)

Israelian, G., et al. 2004, in preparation

Kurucz, R. L. 1993 CDROM, 13, 18

Laird, J. B. 1985, ApJ, 289, 556

Meynet, G., \& Maeder, A. 2002, A\&A, 390, 561

McWilliam, A. 1997, ARA\&A, 35, 503

Molaro, P., \& Pasquini, L. 1994, A\&A, 281, L77

Molaro, P., Bonifacio, P., Castelli, F., \& Pasquini, L. 1997, A\&A, 319, 593

Primas, F., Asplund, M., Nissen, P. E., \& Hill, V. 2000, A\&A, 364, L42

Randich, S., Primas, F., Pasquini, L., \& Pallavicini, R. 2002, A\&A, 387,222

Reeves, H., Fowler, W. A., \& Hoyle, F. 1970, Nature, 226, 727

Ryan, S. G., Norris, J. E., \& Beers, T. C. 1996, ApJ, 471, 254

Sackmann, I.-J., \& Boothroyd, A. I. 1992, ApJ, 392, L71

Salaris, M., \& Weiss, A. 2002, A\&A, 388, 492

Sbordone, L., Bonifacio, P., Castelli, F., \& Kurucz, R. L. 2004, MSAIS, in press

Spite, F., \& Spite, M. 1982, A\&A, 115, 357

Suzuki, T. K., \& Yoshii, Y. 2001, ApJ, 549, 303

Suzuki, T. K., Yoshii, Y., \& Kajino, T. 1999, ApJ, 552, L125

Thévenin, F., Charbonnel, C., de Freitas Pacheco, J. A., et al. 2001, A\&A, 373, 905 (T01)

Travaglio, C., Galli, D., \& Burkert, A. 2001, ApJ, 547, 217

Valle, G., Ferrini, F., Galli, D., \& Shore, S. N. 2002, ApJ, 566, 252

Ventura, P., D’Antona, F., \& Mazzitelli, I. 2002, A\&A, 393, 215 


\section{Online Material}


Table 2. Line list used for the spectrum synthesis in the region of the Be II lines.

\begin{tabular}{|c|c|c|c|c|c|}
\hline $\begin{array}{r}\text { Wavelength } \\
(\mathrm{nm})\end{array}$ & Ion & $\overline{\log g f}$ & Ref. & Pred. & $\begin{array}{r}E \\
\left(\mathrm{~cm}^{-1}\right)\end{array}$ \\
\hline 313.0027 & 107.00 & -2.799 & $\mathrm{~K}$ & & 9182.250 \\
\hline 313.0056 & 40.00 & -0.700 & EM & & 4186.110 \\
\hline 313.0167 & 22.00 & -0.468 & $\mathrm{~K}$ & & 47913.551 \\
\hline 313.0190 & 107.00 & -3.085 & K & $*$ & 9337.492 \\
\hline 313.0191 & 107.00 & -2.657 & $\mathrm{~K}$ & $*$ & 10779.396 \\
\hline 313.0195 & 26.00 & -3.603 & K94 & & 28819.951 \\
\hline 313.0202 & 25.01 & -3.714 & K88 & & 38720.020 \\
\hline 313.0212 & 26.00 & -2.840 & K94 & & 58812.012 \\
\hline 313.0254 & 106.00 & -1.180 & K & & 4205.830 \\
\hline 313.0257 & 23.01 & -0.290 & NBS & & 2808.720 \\
\hline 313.0281 & 108.00 & -1.931 & GG & & 0.000 \\
\hline 313.0290 & 106.00 & -1.136 & K & & 4206.520 \\
\hline 313.0340 & 58.01 & -0.152 & $\mathrm{MC}$ & & 4266.397 \\
\hline 313.0353 & 27.01 & -3.333 & K88 & & 24074.600 \\
\hline 313.0370 & 106.00 & -1.555 & K & & 267.550 \\
\hline 313.0377 & 22.00 & -1.594 & $\mathrm{~K}$ & & 11531.759 \\
\hline 313.0419 & 106.00 & -2.464 & $\mathrm{~K}$ & & 14274.020 \\
\hline 313.0420 & 4.01 & -0.168 & BIE & & 0.000 \\
\hline 313.0439 & 25.00 & -2.518 & K88 & & 30419.609 \\
\hline 313.0476 & 106.00 & -2.450 & $\mathrm{~K}$ & & 14274.970 \\
\hline 313.0519 & 107.00 & -2.701 & K & $*$ & 11114.699 \\
\hline 313.0549 & 25.01 & -1.146 & K88 & & 52373.180 \\
\hline 313.0550 & 107.00 & -3.586 & K & & 6932.594 \\
\hline 313.0562 & 26.01 & -5.213 & K88 & & 30388.543 \\
\hline 313.0569 & 24.01 & -2.457 & K88 & & 42986.621 \\
\hline 313.0570 & 108.00 & -1.559 & GG & & 0.000 \\
\hline 313.0592 & 24.00 & -1.968 & K88 & & 28682.207 \\
\hline 313.0636 & 106.00 & -1.180 & $\mathrm{~K}$ & $*$ & 4188.995 \\
\hline 313.0637 & 25.00 & -1.008 & K88 & & 34423.270 \\
\hline 313.0648 & 106.00 & -1.447 & $\mathrm{~K}$ & & 270.590 \\
\hline 313.0674 & 106.00 & -1.136 & K & $*$ & 4189.812 \\
\hline 313.0726 & 107.00 & -2.851 & $\mathrm{~K}$ & $*$ & 9508.673 \\
\hline 313.0780 & 41.01 & +0.410 & HLB & & 3542.500 \\
\hline 313.0782 & 106.00 & -1.555 & $\mathrm{~K}$ & $*$ & 265.368 \\
\hline 313.0809 & 22.01 & -1.140 & MFW & & 94.100 \\
\hline 313.0813 & 64.01 & -0.083 & $\mathrm{MC}$ & & 9328.864 \\
\hline 313.0823 & 107.00 & -2.670 & $\mathrm{~K}$ & $*$ & 11115.135 \\
\hline 313.0871 & 58.01 & +0.481 & $\mathrm{CC}$ & & 8789.380 \\
\hline 313.0906 & 106.00 & -0.503 & K & $*$ & 18848.234 \\
\hline 313.0910 & 107.00 & -3.018 & $\mathrm{~K}$ & $*$ & 9338.252 \\
\hline 313.0928 & 106.00 & -3.136 & $\mathrm{~K}$ & & 17.770 \\
\hline 313.0932 & 108.00 & -3.358 & GG & & 0.000 \\
\hline 313.1015 & 25.01 & -1.217 & K88 & & 49291.309 \\
\hline 313.1037 & 25.00 & -1.725 & K88 & & 30425.711 \\
\hline 313.1058 & 106.00 & -1.447 & $\mathrm{~K}$ & $*$ & 268.362 \\
\hline 313.1065 & 4.01 & -0.468 & BIE & & 0.000 \\
\hline 313.1070 & 90.01 & -1.559 & $\mathrm{MC}$ & & 0.000 \\
\hline 313.1074 & 106.00 & -0.514 & $\mathrm{~K}$ & $*$ & 18849.781 \\
\hline 313.1109 & 40.00 & -0.400 & $\mathrm{CB}$ & & 4196.850 \\
\hline 313.1110 & 26.00 & -5.171 & K94 & & 24574.652 \\
\hline 313.1115 & 107.00 & -2.627 & $\mathrm{~K}$ & $*$ & 11113.809 \\
\hline 313.1116 & 76.00 & +0.050 & $\mathrm{CB}^{\prime}$ & & 14848.050 \\
\hline 313.1198 & 107.00 & -3.552 & $\mathrm{~K}$ & & 6933.030 \\
\hline 313.1207 & 24.00 & -0.659 & K88 & & 25106.299 \\
\hline 313.1243 & 26.00 & -4.193 & K94 & & 17550.180 \\
\hline 313.1255 & 69.01 & +0.280 & AS & & 0.000 \\
\hline 313.1269 & 107.00 & -3.191 & $\mathrm{~K}$ & $*$ & 9194.144 \\
\hline 313.1326 & 27.01 & -4.088 & K88 & & 17771.711 \\
\hline 313.1339 & 21.01 & -2.430 & K88 & & 59528.422 \\
\hline 313.1395 & 26.01 & -3.656 & K88 & & 30764.484 \\
\hline
\end{tabular}

